\title{
Objectiviser le subjectif pour l'imposer à l'autre : la « normativisation » de contenus évaluatifs comme procédé de modélisation argumentative de l'information sur Internet
}

\author{
Objectivizing the subjective to impose it on the other: \\ the "normativization" of evaluative content as a method \\ of argumentative modeling of information on the Internet
}

\author{
Katarzyna Wołowska \\ Université Catholique Jean Paul II de Lublin \\ wolowska@kul.lublin.pl
}

\begin{abstract}
The aim of the article is to describe some semantic and argumentative mechanisms of Internet discourse processes whose role is to objectify the subjective evaluative judgments contained in the text so as to impose them as conforming to the socially accepted norm. The analyses are based on examples from French news websites available on the Internet.
\end{abstract}

Keywords: evaluation, objective, subjective, linguistic norm, Internet discourse, argumentation, semantic isotopy.

\section{PRÉLIMINAIRES : LE DISCOURS ET LA NORME}

Quel que soit le contexte d'usage de la langue, celle-ci - en tant qu'outil de la communication interhumaine - apparait comme un répertoire de moyens verbaux communs, partagés par la communauté linguistique qui s'en sert. Ce principe traditionnellement reconnu, devenu déjà une banalité, vaut pour toutes les couches de la langue en impliquant, sur le plan du signifié saussurien, différentes composantes de la signification lexicale qui définissent, d'une manière plus ou moins importante, le 
sens véhiculé en contexte. En fait, les conventions d'usage, i.e. l'ensemble de normes socialement admises qui déterminent l'emploi d'une langue, non seulement régissent et modélisent la communication, mais elles vont jusqu'à conditionner à un haut degré son efficacité.

L'espace normatif (normes d'usage de la langue) compte parmi les plus importantes dimensions de la réalité linguistique ; la norme assume une fonction régulatrice par rapport aux oppositions (ou dualités) fortes comme langue/parole (Saussure, 1916), schéma/usage (Hjelmslev, 1971) ou système/parole (Coseriu, 1967, cf. aussi 2001). Dans la théorie de F. Rastier ( $c f$. surtout 2008), qui adopte une perspective interprétative et textuelle, la norme occupe une place importante ; elle n'est pas une, mais il y en a plusieurs, de différents types et de différents niveaux, et elles jouent un rôle crucial dans la description linguistique ${ }^{1}$.

En ce qui concerne la norme relative aux jugements et aux valorisations des contenus sémantiques (ou, si l'on préfère, la norme « axiologique» ou «morale »), elle n'a qu'apparemment le caractère extralinguistique : la composante évaluative étant profondément inscrite dans la langue (signification du lexique), elle l'est aussi en discours, bien qu'à un degré différent selon le cas. En effet, la valorisation du contenu sémantique dans l'usage peut être purement contextuelle (il s'agirait alors d'une sorte d' «hapax évaluatif»), mais elle peut aussi s'appuyer sur une opinion commune, renvoyer à une doxa (cf. par ex. Plantin, 2002, pp. 197-198 ; Amossy, 2000, p. 90 ; Paveau, 2006, pp. 28-30), à une norme socialement admise (ou présentée comme telle), qu'elle soit univoquement acceptée ou qu'elle éveille des controverses. Or, une telle norme « doxale » (qui relève d'une tradition, d'une idéologie, d'une religion, etc.) peut être soumise à diverses modifications, brusques ou progressives, qui soit l'adoucissent, soit la restreignent jusqu'à en faire l'objet de manipulations. Bien entendu, la doxa peut être aussi carrément mise en cause, tant par des individus dans leur emploi quotidien de la langue que par des groupes sociaux entiers cherchant à imposer aux autres leur point de vue, incompatible avec ce que l'on considère comme « normal ».

Dans le présent article, nous entendons envisager les mécanismes sémanticodiscursifs de quelques procédés employés sur Internet (nous nous concentrerons surtout sur les textes venant de sites d'information) qui ont pour but d'imposer au destinataire ses propres jugements évaluatifs en s'appuyant sur une "norme» présupposée, présentée comme incontestable, qu'elle soit effectivement attestée ou non. Nous aborderons cette question du point de vue interprétatif, i.e. dans la perspective du destinataire placé devant un message (texte) susceptible de modéliser ses opinions à travers toute une panoplie de moyens sémantiques à visée rhétorique et argumentative. Nous mettrons particulièrement en relief le rôle des isotopies séman-

\footnotetext{
${ }^{1}$ Pour plus de détails sur les rapports entre les dimensions de la réalité linguistique dans différentes théories du XX ${ }^{\mathrm{e}}$ siècle, $c f$. Wołowska (2014, pp. 73-89).
} 
tiques, constituées d'éléments du contenu manifestés syntagmatiquement et responsables de la cohérence sémantique du texte, surtout des isotopies évaluatives, détectables à l'aide d'outils méthodologiques élaborés au sein de la microsémantique interprétative ( $c f$. surtout Rastier, 1987, 2001).

\section{LES ISOTOPIES ÉVALUATIVES : APERÇU THÉORICO- MÉTHODOLOGIQUE}

L'approche microsémantique et interprétative du texte, à laquelle nous entendons nous référer ici, se fonde sur la méthodologie de l'analyse componentielle qui, sans se restreindre au niveau du contenu lexical décontextualisé, permet d'expliquer les phénomènes sémantico-discursifs de niveaux supérieurs (séquence, texte, intertexte) envisagés obligatoirement en contexte ${ }^{2}$. Le concept d'isotopie concerne en principe le phénomène de la cohérence textuelle : il sert à décrire celle-ci à travers l'analyse de la microstructure sémique des unités lexicales employés en contexte (sémèmes). Définie comme une récurrence de sèmes contextuels (classèmes) sur l'axe syntagmatique du discours (Greimas, 1966), une continuité sémantique à travers une séquence (redondance reflétant une cohérence) (Pottier, 1974, p. 326) ou l'itération syntagmatique d'un même sème ( $c f$. Rastier, 1987, pp. 91, 110) ${ }^{3}$, l'isotopie sémantique est une manifestation syntagmatique d'un même élément du contenu récurrent qui s'identifie et s'impose à l'interprète en contexte (par ex. l'itération du sème /humain/ dans 'homme' et 'roseau' employés dans la célèbre maxime pascalienne : L'homme est un roseau, mais c'est un roseau pensant).

L'itération discursive peut impliquer différents types de sèmes; Rastier établit une typologie d'isotopies fondée sur la distinction fondamentale entre les sèmes génériques (marquant l'appartenance d'un sémème à une classe sémantique, par ex. /humain/ pour 'homme' et 'femme') et spécifiques (distinguant les sémèmes entre eux au sein de la même classe). Ainsi, les isotopies qui résultent de la récurrence de sèmes génériques, appelées isotopies génériques, sont de trois types (tout comme les sèmes génériques) : microgénériques (fondées sur la récurrence d'un sème microgénérique qui fait entrer les sémèmes dans un même taxème, i.e. classe sémantique minimale, par ex. /fruit/ dans 'pomme', 'orange', 'framboise', 'myrtille', etc.), mésogénériques (impliquant la récurrence d’un sème mésogénérique qui indexe les

\footnotetext{
${ }^{2}$ Pour Rastier, la contextualisation est un facteur fondamental de l'interprétation et de l'analyse du texte : «du point de vue interprétatif, tout fragment de texte est incomplet, ou plus précisément indéterminé, et ne sortira de son indétermination que par une contextualisation maximale » (Rastier, 1998, p. 103 ; cf. aussi Wołowska, 2014, p. 92).

${ }^{3}$ Proposée par Greimas (1966), la notion d'isotopie est ensuite devenue l'objet de nombreux travaux linguistiques ( $c f$. Pottier, 1974 ; Arrivé, 1973 ; Berrendonner, 1976 ; Kerbrat-Orecchioni, 1976, 1979 ; Rastier , 1987, 2001 ; Groupe $\mu, 1990$, etc.).
} 
sémèmes faisant partie du même domaine sémantique, par ex. /maritime/ dans 'bateau', 'naviguer', 'vigie', 'ancre', 'matelot', etc.) et macrogénériques (liées à la récurrence d'un sème macrogénérique qui fait entrer les sémèmes dans une dimension sémantique, par ex. /animé/ dans 'femme', 'mouche', 'oiseau', 'rat', etc., ou /non animé/ dans 'pierre', 'table', 'montagne', 'verre', etc.). Les isotopies spécifiques, elles, se forment grâce à l'itération de sèmes spécifiques dont le rôle est de singulariser les sémèmes au sein des classes sémantiques données (taxèmes, domaines, dimensions) ${ }^{4}$. Une autre distinction introduite par Rastier, celle entre les sèmes inhérents (appartenant à la signification relativement stable du sémème) et afférents (actualisés en contexte) est secondaire du point de vue typologique, parce qu'il est rare qu'une isotopie, surtout dans des textes plus longs, soit constituée exclusivement de sèmes inhérents ou de sèmes afférents : normalement, elle inclut les occurrences où le sème isotopant change de statut d'un sémème à l'autre.

Les isotopies sémantiques s'affirment à différents paliers (con)textuels : si l'isotopie minimale se constitue au sein d'un syntagme (deux sémèmes comportant au moins un sème réitéré), cet outil s'avère le plus effectif dans l'analyse thématique des textes et des corpus entiers, au niveau tant local que global. L'identification des isotopies au cours de la lecture linéaire du texte s'appuie non seulement sur la signification des unités lexicales à partir de laquelle elles acquièrent un sens particulier en contexte, mais aussi sur la stratégie d'actualisation de sèmes en cours de lecture appelée présomption d'isotopie ( $c f$. Rastier, 1985, p. 34). Il s'agit là d'une hypothèse interprétative validée par le contexte en amont que l'interprète admet a priori pour la suite du texte et qu'il cherche à confirmer ou, éventuellement, à invalider par le contexte en aval. Ce mécanisme vaut aussi bien pour les isotopies «thématiques » (spécifiques, micro- et mésogénériques), les plus responsables de la cohérence sémantique du texte, que pour les isotopies macrogénériques évaluatives qui nous intéresseront ici particulièrement.

\section{L'ESPACE NORMATIF ET LA VALORISATION DU CONTENU SÉMANTIQUE EN DISCOURS : LE CAS DU DISCOURS MÉDIATIQUE}

Grâce à l'existence des normes d'usage de la langue que les locuteurs admettent a priori et instinctivement, on peut déterminer, d'une manière plus ou moins précise, une signification «inhérente » des unités lexicales. Celle-ci, communément admise et codifiée dans les dictionnaires de langue, présente une structure (relativement) stable, aussi bien au niveau de ses composantes définitoires qu'à celui de la valeur axiologique (positive ou négative) qu'on lui assigne. Ainsi, par exemple, les lexèmes comme amour, ordre, tranquillité, etc. sont généralement valorisés positivement (que

\footnotetext{
${ }^{4}$ Rastier cite ici en exemple un vers d'Éluard, L'aube allume la source, où la récurrence du sème spécifique inhérent /inchoatif/ dans 'aube', 'allume' et 'source' forme une isotopie spécifique.
} 
cela se reflète explicitement dans les définitions lexicographiques ou reste implicite), alors que les unités comme mort, désordre, désespoir, etc. apparaissent comme porteuses d'une valeur négative, signalée dans bien des cas par la forme morphologique (préfixes négatifs comme dés-ordre, in-commode, mal-heureux, etc.). Du moment où l'unité lexicale se trouve employée en contexte, celui-ci peut soit valider sa signification inhérente, y compris sa composante évaluative (valorisation positive ou négative), soit l'invalider et la modifier à un degré plus ou moins important.

Dans le cas du contenu évaluatif, la modification consiste habituellement en un renversement de valeurs : ce qui est considéré et généralement admis comme bon, juste, louable, etc. se trouve déprécié, tandis que ce qui «normalement» est jugé mauvais, inacceptable, négatif se trouve valorisé positivement. Un tel procédé de renversement semble fréquent surtout dans les textes littéraires où il devient un moyen esthético-argumentatif employé pour attirer l'attention du destinataire, plus spécifiquement pour le choquer et/ou l'émouvoir. Pourtant, d'autres contextes discursifs deviennent eux aussi propices à l'emploi de ce type de procédé, surtout le discours public (politique ou médiatique).

En fait, vu l'expansion croissante de l'information rapide, l'univers médiatique semble un milieu où la modélisation libre du contenu évaluatif devient particulièrement visible et rhétoriquement efficace. Dans la réalité socio-politique dynamiquement changeante, le contenu évaluatif, de par sa nature précaire et instable face au contexte, devient encore plus fragile et susceptible de manipulations. La portée globale de l'information diffusée permet ainsi d'atteindre, parfois sans beaucoup d'effort, l'effet dont rêvaient les orateurs romains : celui de mener les foules, de les influencer en leur imposant (d'une manière plus ou moins implicite) le point de vue précis. L'objectivisation discursive à travers la «normativisation » d'un point de vue subjectif et l'introduction de celui-ci dans l'espace interpersonnel, voire public, associable partiellement à ce qu'on appelle couramment manipulation, est un phénomène connu et pratiqué depuis toujours. Pourtant, la nouvelle forme de la communication globalisante, qui permet d'influencer les masses via les média et le réseau Internet, ouvre la possibilité de modifier ou même d'abolir les normes existantes pour en créer d'autres, parfois précisément contraires (surtout sur le plan des valeurs axiologiques assignées à différentes attitudes et activités de l'homme contemporain).

\section{L'OBJECTIVISATION DU SUBJECTIF ET LA NORME IMPOSÉE : QUELQUES EXEMPLES D'UNE MODÉLISATION ARGUMENTATIVE DE L'INFORMATION SUR INTERNET}

Pour illustrer le phénomène mentionné plus haut, nous nous concentrerons sur un seul mode de communication massive, à savoir sur le discours d'Internet. Nous prendrons en compte le cas particulier de l'information sur la politique migratoire 
européenne des années 2015-2017 qui, très présente dans les médias, éveille des controverses et divise les politiciens et les sociétés. Ce problème d'actualité, crucial du point de vue politique vu son grand impact sur la structure de la société européenne et les conditions de vie des citoyens, se trouve véhémentement discuté dans l'espace public par les partisans et les opposants de la politique d'ouverture des frontières. Objectivement parlant, les arguments avancés par les deux côtés devraient permettre une discussion constructive et équilibrée ; cependant, il n'est pas rare que le discours produit à ce sujet regorge d'émotions extrêmes, exprimées à travers un vocabulaire axiologiquement marqué.

\subsection{LES CONTRAINTES NORMATIVES LIÉES À LA FORME DU DISCOURS}

Comme d'habitude dans de tels cas, la discussion se déroule sur deux principaux modes : officiel et privé. La forme de ces deux discours diffère nettement, le discours en privé étant naturellement beaucoup plus libre et spontané, à un vocabulaire moins recherché, sinon familier. Ce qui semble ici fondamental, c'est la question de l'anonymat propre à la communication par Internet. La possibilité de communiquer sans être reconnu permet non seulement d'exprimer sans ambages ses opinions et ses émotions, mais aussi de modéliser (ou même de fausser) à volonté son image virtuelle et son identité dans le Réseau ; l'anonymat est ainsi susceptible de rendre le locuteur d'un côté plus courageux, de l'autre plus méchant et plus agressif.

Il en va autrement du discours officiel, surtout celui des politiciens et de la diplomatie, qui se trouve soumis à des règles très strictes et qui exige une grande prudence au niveau de l'expression verbale. En ce qui concerne le discours médiatique (presse, radio, télévision, ainsi que les versions électroniques des articles de journaux et les matériaux audio-visuels publiés sur Internet), il apparaît comme une forme « intermédiaire » : sans pouvoir profiter de tous les privilèges de la conversation en privé, il permet au locuteur de présenter très ouvertement ses opinions dans l'espace public. La forme qui nous intéresse ici, ce sont les articles d'actualité publiés sur les sites d'information; il convient pourtant de mentionner aussi dans ce contexte les blogs et les films d'opinion publiés sur Youtube dont la portée et l'impact sur l'opinion publique sont très importants.

\subsection{L'OBJECTIF ET LE SUBJECTIF : UNE MISE AU POINT TERMINOLOGIQUE}

La question de la subjectivité dans le langage ( $c f$. surtout Benveniste, 1966; Kerbrat-Orecchioni, 1980) occupe en linguistique une place importante, vu que, « toute unité lexicale est, en un sens, subjective, puisque les 'mots' de la langue ne 
sont jamais que des symboles substitutifs et interprétatifs des 'choses' » (KerbratOrecchioni, ibidem, p. 79). Cependant, l'acception du terme 'subjectif 'que nous entendons employer ici est à la fois plus spécifique et plus étendue dans la mesure où, d'un côté, elle se réfère à l'opinion à caractère socio-politique et, de l'autre, s'extrapole d'un individu (présupposé dans la première acception du terme) à une collectivité dont les membres partagent les mêmes convictions. Il s'agit donc d'une subjectivité collective, propre à un groupe social ou à un groupe d'intérêts, exprimée publiquement d'une manière ferme, voire agressive. Elle correspond à une attitude rigide que, dans certains cas extrêmes, on pourrait même appeler fanatique : aveugle face aux faits, interprétant les événements d'une façon tendancieuse (non objective), rejetant a priori tout argument non conforme au point de vue adopté.

Une telle radicalisation d'opinions et de leur expression verbale caractérise les conflits publics les plus fervents, surtout ceux qui se trouvent suffisamment «martelés » dans les médias; dans le cas qui nous intéresse, celui de la politique migratoire européenne, ce phénomène concerne aussi bien les partisans que les opposants de la migration. Contrairement aux apparences, il s'agit là d'un conflit de deux points de vue subjectifs qui, bien que partagés l'un et l'autre par des groupes importants d'individus, ne sauraient pour cette seule raison devenir objectifs, alors que le groupe donné s'efforce de les imposer comme tels en procédant à leur objectivisation et/ou leur «normativisation » en discours.

\subsection{LA POLITIQUE ANTI-MIGRATOIRE POLONAISE ET HONGROISE FACE À LA POLITIQUE EUROPÉENNE : ANALYSES}

Pour bien saisir le mécanisme qui nous intéresse, nous envisagerons quelques exemples venant de sites d'information français, concernant la politique de la Pologne et de la Hongrie. L'attitude réservée des deux pays face à l'accueil des migrants éveille en effet de vives émotions dans l'espace public, ce qui se reflète au niveau sémantique dans la construction des faisceaux isotopiques à caractère évaluatif. Ainsi, dans les exemples analysés plus loin, l'isotopie macrogénérique /négatif/ accompagne très régulièrement les isotopies de niveaux inférieurs (méso- et microgénériques) en contribuant à esquisser une image non favorable de la politique critiquée, ainsi qu'à déprécier les politiciens qui en sont responsables. Voici un texte publié après la victoire du Parti Droit et Justice polonais dans les législatives de 2015 :

(1) Le Parti Droit et Justice (PiS), ou la Pologne anti-migrants

Après avoir installé à la Présidence, pour cinq ans, le jeune juriste Andrzej Duda, le Parti ultraconservateur Droit et Justice (PiS) de Jaroslaw Kaczynski devrait remporter haut la main les législatives du dimanche 25 octobre. De quoi bientôt disputer à la Hongrie le rôle de trouble-fête de l'Union européenne? 
On savait très conservateur, nationaliste, climato-sceptique et antieuropéen le nouveau chef de l'Etat polonais, Andrzej Duda, 43 ans, élu à la surprise générale en mai dernier avec $51,55 \%$ des voix (...).

Le jeune président avait réservé sa première visite d'Etat à l'Estonie, et réclamé $\underline{\text { à grands cris une base de l'Otan en Pologne. Puis, battant froid à son concitoyen le prési- }}$ dent du Conseil européen Donald Tusk, issu d'un parti concurrent, il s'est rué à Londres pour apporter son soutien eurosceptique à David Cameron.

Mais à l'approche de la présidentielle du 25 octobre, l'héritier spirituel du défunt président Lech Kaczynski, tué dans le crash de Smolensk, en 2010, a montré une facette encore plus radicale et cynique, en agitant sans vergogne la carte anti-émigrés pour gagner des suffrages. Ainsi, après avoir sans grand succès répété à qui voulait l'entendre que la Pologne n'avait pas les moyens d'accueillir 7000 des 120.000 réfugiés que doit se répartir l'UE, voici que ce catholique pratiquant martèle qu'il faut «protéger les Polonais » contre les « éventuelles épidémies » dont seraient porteurs les migrants.

Duda ne faisait que reprendre les propos de son mentor Jaroslaw Kaczynski, fondateur du parti PiS (Droit et Justice), qui dénonçait le " choléra qui sévit déjà sur les îles

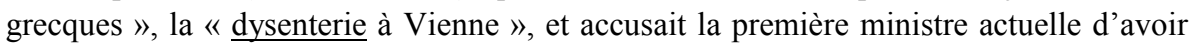
«signé un accord secret avec Bruxelles pour accueillir 100.000 musulmans ». Et prédisant à la Pologne «la dévastation des églises, l'introduction de la sharia, l'obligation d'adopter l'islam et le terrorisme ». Un langage que ne désavouerait pas le premier ministre hongrois Viktor Orban... (https://www.marianne.net/monde/le-partidroit-et-justice-pis-ou-la-pologne-anti-migrants).

Le texte (1) concerne surtout le Président de la Pologne, mais il se réfère à la politique polonaise en général ; celle-ci s'y trouve peinte d'une manière décidément négative. Les principales isotopies qui s'établissent au niveau aussi bien macrogénérique (isotopie évaluative dominante) que méso- et microgénérique (responsables de la thématique du texte) et spécifique présentent l'ordre suivant :

\begin{tabular}{|l|l|l|l|}
\hline \multicolumn{1}{|c|}{ Classes sémantiques } & \multicolumn{3}{c|}{ ISOTOPIES } \\
\hline $\begin{array}{l}\text { dimension } \\
\text { (niveau macrogénérique) }\end{array}$ & /négatif/ & \\
\hline $\begin{array}{l}\text { domaine } \\
\text { (niveau mésogénérique) }\end{array}$ & /politique/ & \\
\hline $\begin{array}{l}\text { taxème } \\
\text { (niveau microgénérique) }\end{array}$ & /nationaliste/ & /anti-européen/ & /anti-migrant/ \\
\hline traits sémaniques spécifiques & /radical/ & $\begin{array}{l}\text { /faux/ } \\
\text { /fanatique/ }\end{array}$ & /hystérique/ \\
\hline
\end{tabular}

L'isotopie évaluative /négatif/ qui détermine l'effet pragmatique du texte fait partie du même faisceau isotopique que la récurrence des traits génériques /nationaliste/ ('le Parti ultraconservateur Droit et Justice', 'conservateur', 'natio- 
naliste', 'protéger la Pologne', etc.), /anti-européen/ ('trouble-fête de l'Union européenne', 'climatosceptique et antieuropéen', 'battant froid à son concitoyen le président du Conseil européen Donald Tusk', 'soutien eurosceptique', etc.) et /antimigrant/ ('la Pologne anti-migrants', 'la carte anti-émigrés', 'les «éventuelles épidémies » dont seraient porteurs les migrants', etc.). Ces isotopies génériques clés sont accompagnées de récurrences sémiques plus spécifiques, relatives aux émotions et attitudes attribuées aux politiciens en question, à savoir /radical/ et /fanatique/ ou même /hystérique/ ('facette encore plus radicale et cynique', 'réclamé à grands cris', 'il s'est rué à Londres', 'prédisant à la Pologne «la dévastation des églises, l'introduction de la sharia, l'obligation d'adopter l'islam et le terrorisme »', 'qui dénonçait le «choléra qui sévit déjà sur les îles grecques", la «dysenterie à Vienne »', etc.), traits qui sont particulièrement mal vus dans le contexte de la politique, surtout internationale. À tout cela s'ajoute le trait/faux/ suggéré par un renvoi à l'enseignement de l'Église catholique auquel 'ce catholique pratiquant' s'oppose par ses actes.

Une telle image négative des politiciens, servant à déprécier la politique qu'ils réalisent, n'a rien de surprenant dans ce genre de discours (critique politique) : il est naturel que l'auteur soit libre de présenter son point de vue d'une manière aussi directe et émotionnelle. Ce qui nous intéresse particulièrement, c'est l'aspect pragmatico-argumentatif de ce texte, plus précisément, la façon dont la valeur négative assignée à la politique de la Pologne se trouve construite pour s'imposer au lecteur. En effet, derrière l'emploi du vocabulaire à valeur négative et discréditante, nous pouvons observer ici un échantillon d'au moins quatre procédés d'objectivisation du contenu évaluatif véhiculé dans ce texte à tout point exemplaire, à savoir :

a) renvoi à un point de repère positif (situé à l'antipode de ce qu'on déprécie) : ici, c'est la politique européenne pro-migrant ;

b) analogie à connotation négative déjà établie et reconnue (ici Kaczyński comparé à Orbán, le Président hongrois dont l'attitude très réservée envers les migrants est bien connue) ;

c) le choix de citations qui, d'un côté, rendent l'information plus vraisemblable et, de l'autre, aident à présenter le politicien cité comme agressif et repoussant ( $c f$. « choléra qui sévit déjà sur les îles grecques », « la dysenterie à Vienne »);

d) renvoi à une autorité, surtout celle de l'Église catholique et du Pape (ici, il s'agit de souligner l'hypocrisie d'un «catholique pratiquant» qui refuse d'accueillir les migrants).

Nous ne saurions décrire ici tous ces procédés, qui mériteraient d'ailleurs une analyse exhaustive et approfondie ; aussi, nous concentrons-nous sur la façon dont fonctionnent dans de tels contextes les isotopies sémantiques évaluatives. Le mécanisme principal du processus d'objectivisation des contenus évaluatifs (/positif/ vs /négatif/) véhiculés dans un texte s'appuie sur le renvoi, plus ou moins explicite, à un point de repère à valeur opposée (surtout positive) que l'on présente comme 
une norme pour mieux justifier ses opinions et actes ou pour mieux critiquer ceux des autres. Un tel mécanisme se laisse observer assez nettement dans le discours sur la politique européenne pro-migrant des années 2015-2017 qui, très appréciée dans les média du mainstream, y est présentée habituellement comme évidente, naturelle et incontestablement positive, tant pour la société de l'Union européenne en général que pour les nations des États-membres en particulier. Les images de migrants sauvés à la mer ou chaleureusement accueillis sur les gares par des foules enthousiastes, images multipliées et vivement commentées dans l'espace médiatique (télé, journaux, Internet), ont pour objectif de renforcer l'identité communautaire des Européens, de les rendre fiers de faire partie d'une société généreuse et accueillante qui sait aider les faibles, mais aussi - ce qui est non moins important - de les convaincre que la politique réalisée par les autorités est correcte et avantageuse pour tout le monde. Les démarches pro-migrant entreprises par les politiciens deviennent ainsi dans la conscience collective de la société européenne un point de référence positif, une norme idéologique et même axiologique que la réitération inlassable dans l'espace public rend de plus en plus incontestable.

Il faut souligner néanmoins que l'intérêt et l'efficacité d'un tel discours argumentatif s'appuient habituellement sur un vif jeu d'oppositions et sur un raisonnement à caractère dialectique; le processus d'imposer aux masses une norme d'opinion exige en fait l'existence d'un volet opposé, un exemple négatif qui assure à la création médiatique un sain équilibre en mettant mieux en relief la valeur positive du « politiquement correct ». Pour obtenir cet effet, il faut que l'opposition entre les deux pôles, positif et négatif, soit suffisamment marquée et radicale, d'où les textes comme celui que nous venons d'analyser plus haut. Au niveau linguistique, cette opposition radicalisée entre les contenus à valeur positive et négative se traduit par l'emploi de moyens lexicaux et sémantiques spécifiques, tels que le vocabulaire évaluativement marqué (utilisé surtout pour esquisser les images négatives), les figures du discours comme l'hyperbole (et d'autres procédés d'intensification), l'ironie ou le sarcasme, ainsi que de nombreux marqueurs à caractère évaluatif et émotionnel. L'établissement d'une opposition bien lisible assume en effet une fonction à la fois catalysatrice (neutraliser ou même ridiculiser les convictions contraires) et confirmatrice (souligner la supériorité des convictions présentées comme correctes).

Dans le cas qui nous intéresse ici, la politique anti-migrant polonaise et hongroise sert ainsi d'exemple négatif, elle représente l'attitude non désirable et incorrecte qui met d'autant mieux en lumière la norme imposée. Cette image fortement négative des deux pays se manifeste déjà dans les titres de textes publiés à ce propos sur Internet, aussi bien sur les sites officiels des journaux que sur des blogs ou sur de moindres sites d'information. En voilà quelques exemples qui renvoient (directement ou plus implicitement) au sujet analysé ; le choix même du vocabulaire employé dans ces titres ( $c f$. les éléments soulignés) signale la valeur /négatif/ assignée à la politique hongroise et polonaise au sein des textes entiers. 
(2) Sentiment anti-immigrant en Pologne : pure haine ou violence éphémère? (http://www. middleeasteye.net/fr/reportages/sentiment-anti-immigrant-en-pologne-pure-haine-ouviolence-ph-m-re-599622740)

(3) Pologne: "La haine et le racisme ne sauraient être expliqués par l'histoire, ni par rien d'autre " (http://www.telerama.fr/monde/la-haine-et-le-racisme-ne-sauraient-etreexpliques-par-1-histoire-ni-par-rien-d-autre,133477.php)

(4) En Pologne, la peur des immigrés imaginaires (http://www.liberation.fr/planete/ 2015/10/21/en-pologne-la-peur-des-immigres-imaginaires_1407896)

(5) La Hongrie rétablit la détention systématique des migrants, contraire au droit européen (http://www.france24.com/fr/20170307-hongrie-orban-detention-systematiquemigrants-serbie-croatie-union-europeenne)

(6) Hongrie : une consultation anti-européenne et anti-migrants lancée (http://www.rfi.fr/ europe/20170402-hongrie-consultation-anti-europeenne-lancee-refugies-immigration)

(7) Demandes d'asile : pourquoi la Hongrie et la Pologne défient les règles de l'UE (http://www.lexpress.fr/actualite/monde/europe/pourquoi-la-hongrie-et-la-pologne-sepermettent-de-defier-les-regles-de-l-ue_1917833.html)

(8) Le sinistre bilan des droites extrêmes au pouvoir (http://www.humanite.fr/le-sinistrebilan-des-droites-extremes-au-pouvoir-635573)

Les exemples (2), (3) et (4) renvoient à des émotions ou attitudes négatives, nommées explicitement dans les titres et confirmées dans le contenu total des articles, telles que la haine, la violence, le racisme ou la peur fausse et infondée. $\mathrm{Vu}$ qu'il s'agit là de sentiments forts et réprouvables, le destinataire du texte est invité dès le début à se forger une opinion négative à propos de la nation à laquelle ces attitudes envers les migrants se trouvent assignées. Une référence à la norme positive contraire (tolérance, ouverture, compassion), associée à d'autres sociétés de l'Union européenne, est ici implicite mais tout à fait lisible; en revanche, elle se trouve exprimée plus explicitement dans les exemples $(5),(6)$ et (7) où le droit européen est présenté comme un point de référence incontestable et positif ( $c f$. la valorisation contextuelle des lexèmes : 'détention', 'contraire', 'anti-européenne', 'anti-migrants', 'défier' => /négatif/ vs 'demandes d'asile', 'droit', 'règles', 'européen' => /positif/). Enfin, le titre (8) illustre une autre tendance, très présente dans le discours médiatique relatif à la Pologne et à la Hongrie ( $c f$. le texte 1$)$, consistant à réprouver toute manifestation de nationalisme, communément reproché à l'extrême droite (infiniment diabolisée dans l'espace public, non seulement francophone) et associé à des valeurs et attitudes fortement négatives (haine, fanatisme, fermeture, racisme, intolérance, etc.). Or, objectivement parlant, la définition lexicographique du lexème nationalisme (théorie politique qui affirme la prédominance de l'intérêt national par rapport aux intérêts des classes et des groupes qui constituent la nation ou par rapport aux autres nations de la communauté internationale, $D L^{5}$ ) ne signale aucu-

\footnotetext{
${ }^{5}$ Dictionnaire Larousse en ligne (DL), http://www.larousse.fr/dictionnaires/francais (consulté le 04.08.2017).
} 
nement de tels traits sémantiques à valeur négative. L'image repoussante de la politique nationale (ou « nationaliste ») semble refléter plutôt les convictions subjectives d'un groupe qui cherche à les imposer aux autres, à les rendre incontestables dans la conscience collective de la société, bref, à les normativiser en opposant la «mauvaise » extrême droite nationaliste à la « bonne » politique internationale d'ouverture et de tolérance.

\section{EN GUISE DE CONCLUSION}

Les procédés rhétorico-argumentatifs que nous venons d'analyser sont employés régulièrement dans le discours politique et journalistique, sur les sites d'information et les blogs publiés sur Internet. Pour influencer le lecteur, l'énonciateur recourt à différentes stratégies qui renforcent le jugement évaluatif et permettent de le présenter comme correct et incontestable, telles que l'emploi de marqueurs d'émotions fortes, l'hyperbolisation et le renvoi à d'autres procédés d'intensification et d'exagération ou la mise en place d'oppositions sémantiques à valeur évaluative (/bon/ vs /mauvais/, /acceptable/ vs /inacceptable/, /positif/ vs /négatif/); ces dernières s'établissent à travers des faisceaux isotopiques adéquats qui se manifestent aux niveaux textuel et intertextuel. Certes, les mécanismes sémantico-discursifs décrits ne sont pas strictement spécifiques de l'objectif pragmatique dont nous venons de parler (influencer l'opinion publique à travers l'objectivisation de contenus évaluatifs véhiculés dans le texte); pourtant, ils participent efficacement dans la réalisation de cet objectif en accompagnant la stratégie centrale, celle de renvoyer à un point de repère positif, représenté ici par la politique européenne pro-migrant. 


\section{BIBLIOGRAPHIE}

Amossy, R. (2000). L'argumentation dans le discours. Paris : Nathan.

Arrivé, M. (1973). Pour une théorie des textes polyisotopiques. Langages, 31, pp. 53-63. DOI: 10.3406/lgge.1973.2235.

Benveniste, E. (1966). Problèmes de linguistique générale. Paris : Gallimard.

Berrendonner, A. (1976). De quelques aspects logiques de l'isotopie. Linguistique et sémiologie, 1, pp. 117-135.

Coseriu, E. (1967). Teoría de lenguaje y lingüistica general : cinco estudios. Madrid : Gredos.

Coseriu, E. (2001). L'homme et son langage. Louvain-Paris : Peeters.

Greimas, A. J. (1966). Sémantique structurale. Paris : Larousse.

Groupe $\mu$ (1990). Rhétorique de la poésie. Paris : Seuil.

Hjelmslev, L. (1971). Essais linguistiques. Paris : Minuit.

Kerbrat-Orecchioni, C. (1976). Problématique de l'isotopie. Linguistique et sémiologie, 1, pp. 11-33.

Kerbrat-Orecchioni, C. (1979). De la sémantique lexicale à la sémantique de l'énonciation. Lille: Service de reproduction des thèses.

Kerbrat-Orecchioni, C. (1980). L'énonciation. De la subjectivité dans le langage. Paris : Armand Colin.

Paveau, M.-A. (2006). Les prédiscours. Sens, mémoire, cognition. Paris : Sorbonne.

Plantin, Ch. (2002). Doxa. In P. Charaudeau, D. Maingueneau, Dictionnaire de l'analyse du discours (pp. 197-198). Paris : Seuil.

Pottier, B. (1974). Linguistique générale. Théorie et description. Paris : Klincksieck.

Rastier, F. (1985). L'isotopie sémantique, du mot au texte. Thèse de Doctorat d'État. Paris : Université de Paris IV.

Rastier, F. (1987). Sémantique interprétative. Paris : PUF.

Rastier, F. (1998). Le problème épistémologique du contexte et le statut d'interprétation dans les sciences du langage. Langages, 129, pp. 97-111. DOI : 10.3406/lgge.1998.2149.

Rastier, F. (2001). Arts et sciences du texte. Paris : PUF.

Rastier, F. (2008). Conditions d'une linguistique des normes. Texto!, XVIII, 3. Retrieved from http://www.revue-texto.net/docannexe/file/1612/rastier_normes.pdf (consulté le 02.09.2017).

Saussure, F. de (1916 [éd. crit. 1972]). Cours de linguistique générale. Paris : Payot.

Wołowska, K. (2014). Le sens absent. Approche microstructurale et interprétative du virtuel sémantique. Frankfurt : Peter Lang. 